\title{
A relação entre a autoeficácia e o autoconceito em jovens atletas de futebol: uma
}

\section{revisão de escopo}

\author{
The relationship between self-efficacy and self-concept in Young soccer athletes: a scope review \\ La relación entre la autoeficacia y el autoconcepto em jóvenes deportistas de fútbol: una revisión \\ del alcance
}

Recebido: 14/01/2022 | Revisado: 18/01/2022 | Aceito: 21/01/2022 | Publicado: 23/01/2022

\author{
Willian Henrique Corrêa \\ ORCID: https://orcid.org/ 0000-0003-4490-125X \\ Universidade Federal do Paraná, Brasil \\ willianhcorrea@hotmail.com \\ Adair José Pereira da Rocha \\ ORCID: https://orcid.org/0000-0002-3308-121X \\ Universidade Federal do Paraná, Brasil \\ E-mail adairbasquetecuritiba@gmail.com \\ Gilson Brun \\ ORCID: https://orcid.org/0000-0002-9090-0736 \\ Universidade Federal do Paraná \\ gilsonbrun1@gmail.com \\ Gislaine Cristina Vagetti \\ ORCID: https://orcid.org/0000-0003-0704-1297 \\ Universidade Estadual do Paraná, Brasil \\ E-mail: gislainevagetti@ hotmail.com \\ Valdomiro de Oliveira \\ ORCID: https://orcid.org/0000-0002-8709-8471 \\ Universidade Federal do Paraná, Brasil \\ E-mail: oliveirav457@gmail.com
}

\begin{abstract}
Resumo
Objetivo: investigar estudos encontrados no meio científico/acadêmico que relacionem a autoeficácia ou o autoconceito com jovens atletas de futebol. Método: A revisão de escopo foi realizada utilizando-se da metodologia elaborada pelo Instituto Joanna Briggs (JBI) e inclui estudos em plataformas de pesquisa. Foram considerados estudos publicados em inglês, espanhol, português, utilizando um limite de data de 10 anos nas pesquisas, literatura cinzenta, teses e dissertações. A estratégia de busca seguiu o processo natural de rastreio de documentos, com a pesquisa feita nas seguintes bases de dados: Periódicos CAPES, BVS, SportDiscus, Eric, Pepsic e PsychINFO. Foram usados os descritores "self-efficacy", "self-concept", "soccer", "young athletes" e "collective sports". Resultado: Foram selecionados 11 artigos, sendo estes de interesse na relação da autoeficácia com jovens atletas de futebol ou relacionados a outra modalidade esportiva. Conclusão: Nestes artigos, encontramos uma série de pesquisas que possuem metodologias diferentes para explorar estas questões. Interessante notar que os estudos envolvem vários elementos que fazem parte do contexto esportivo, como por exemplo: a família e os treinadores, peças chaves para o sucesso desportivo do atleta. Os trabalhos não possuem uma forma de investigação única, o que mostra como este tema pode ser submetido a várias formas de análise e pesquisado de maneira diversa. Após o processo de seleção e análise, não foram encontrados estudos que relacionem o autoconceito com jovens atletas de futebol.
\end{abstract}

Palavras-chave: Autoeficácia; Autoconceito; Futebol; Jovens atletas.

\begin{abstract}
Objective: to investigate studies found in the scientific/academic environment that relate self-efficacy or self-concept with young soccer athletes. Method: The scope review was carried out using the methodology developed by the Joanna Briggs Institute (JBI) and includes studies on research platforms. Studies published in English, Spanish, Portuguese were considered, using a 10-year date limit on surveys, gray literature, theses and dissertations. The search strategy followed the natural process of document tracking, with the search carried out in the following databases: CAPES, BVS, SportDiscus, Eric, Pepsic and PsychINFO journals. The descriptors "self-efficacy", "self-concept", "soccer", "young athletes" and "collective sports" were used. Result: Eleven articles were selected, these being of interest in the relationship of self-efficacy with young soccer athletes or those related to another sport. Conclusion: In these articles, we find a series of studies that have different methodologies to explore these questions. It is interesting to note that the studies involve several elements that are part of the sporting context, such as: family and coaches, key players for the athlete's sporting success. The works do not have a single form of investigation, which shows how this
\end{abstract}


topic can be subjected to various forms of analysis and researched in different ways. After the selection and analysis process, no studies were found that relate self-concept to young soccer athletes.

Keywords: Self-efficacy; Self-concept; Football; Young athletes.

\begin{abstract}
Resumen
Objetivo: investigar estudios encontrados en el medio científico/académico que relacionen la autoeficacia o el autoconcepto con jóvenes deportistas de fútbol. Método: La revisión de alcance se realizó utilizando la metodología desarrollada por el Instituto Joanna Briggs (JBI) e incluye estudios sobre plataformas de investigación. Se consideraron estudios publicados en inglés, español, portugués, con fecha límite de 10 años en encuestas, literatura gris, tesis y disertaciones. La estrategia de búsqueda siguió el proceso natural de seguimiento de documentos, con la búsqueda realizada en las siguientes bases de datos: revistas CAPES, BVS, SportDiscus, Eric, Pepsic y PsychINFO. Se utilizaron los descriptores "autoeficacia", "autoconcepto", "fútbol", "atletas jóvenes" y "deporte colectivo". Resultado: Se seleccionaron once artículos, siendo estos de interés en la relación de la autoeficacia con jóvenes deportistas de fútbol o relacionados con otro deporte. Conclusión: En estos artículos encontramos una serie de estudios que tienen diferentes metodologías para explorar estas cuestiones. Es interesante notar que los estudios involucran varios elementos que forman parte del contexto deportivo, tales como: la familia y los entrenadores, actores claves para el éxito deportivo del atleta. Los trabajos no tienen una única forma de investigación, lo que muestra cómo este tema puede ser sometido a diversas formas de análisis e investigado de diferentes maneras. Luego del proceso de selección y análisis, no se encontraron estudios que relacionen el autoconcepto de los jóvenes deportistas de fútbol.
\end{abstract}

Palabras clave: Autoeficacia; Autoconcepto; Fútbol americano; Atletas jóvenes.

\title{
1. Introdução
}

A autoeficácia (AE) trata acerca da capacidade da pessoa de alcançar determinado rendimento. Segundo Bandura (1997, p.3), a "autoeficácia percebida se refere às crenças de um indivíduo em sua capacidade em organizar e executar cursos de ação requeridos para produzir certas realizações ou feitos”. Para (Bronfenbrenner \& Morris, 2007), a autoeficácia é uma característica que é estimulada pelo meio, mas construída pelo próprio sujeito.

Neste sentido, compreendemos a autoeficácia como a convicção de alguém em realizar uma tarefa na qual se julga, de forma subjetiva, capaz de reagir com êxito à realidade imposta a si (Reverdito, 2016). Os sentimentos de eficácia se baseiam em seis fontes de informações: Realização de Desempenho, Experiências Indiretas, Persuasão Verbal, Experiências Imaginativas, Estados Fisiológicos e Estados Emocionais. Dependendo do ambiente exposto, uma pode ser mais influente que a outra (Kyrillos, 2016).

A autoeficácia está estritamente ligada aos aspectos psicológicos e físicos, além da relação de ambos com o ambiente físico, uma vez que trabalha com a capacidade do indivíduo de realizar tarefas (Bandura, 1997). Devido a essa conexão dos fatores psicossociais com a convicção de ter realizado uma atividade com êxito, há relação com a iniciação esportiva em jovens (Matias et al., 2009).

O esporte vem a ser um instrumento de desenvolvimento da autoeficácia (Gallagher, 2012; Coalter, 2013), visto que a cada desafio proposto o adolescente tem como objetivo resolvê-lo e não evitá-lo. Para (Coalter, 2013), durante a prática física o indivíduo é exposto a situações a partir das quais ele vem a desenvolver habilidades para sanar o problema da forma mais eficaz possível, além de adquirir autorregulação, isto é, aprender a lidar com outras pessoas e outros desafios propostos. Nesse sentido, as temáticas deste estudo nos auxiliam a refletir a importância destes aspectos.

Autoconceito (AC) é um termo que vem sendo estudado há algumas décadas, devido à sua grande influência no desenvolvimento humano em relação às suas atuações perante os grupos sociais inseridos ou simplesmente em relação a si mesmo (SErra, 1988; Stevanato et al., 2003).

$\mathrm{O}$ autoconceito é considerado uma avaliação do indivíduo face às suas características pessoais e à sua competência comportamental, ou seja, como ele se vê em relação a um ideal que ele mesmo almeja (Mccullough et al., 2000). Essa construção do autoconceito sofre a interferência de quatro aspectos: 1) o modo como as outras pessoas observam o indivíduo; 2) a noção que o indivíduo guarda do seu desempenho em situações específicas; 3) o confronto da conduta da pessoa com a 
dos pares sociais com os quais se encontra identificada; 4) a avaliação de um comportamento específico em função de valores veiculados por grupos normativos.

Além dos quatro aspectos citados acima, o AC pode ser analisado por cinco diferentes fatores, sendo eles: Acadêmico, Social, Emocional, Familiar e Físico. Desta forma, é possível avaliar qual contexto do autoconceito necessita de maiores cuidados, possibilitando assim as intervenções necessárias (Monteiro, 2012; Sarriera et al., 2015).

A prática regular da atividade física, segundo Tamayo et al (2001), contribui de forma positiva para a construção do AC. Nesse estudo, verificou-se que os praticantes de exercício físico obtiveram os maiores escores para o AC.

$\mathrm{O}$ esporte, quando oportunizado e atrativo para as crianças, contribui para o desenvolvimento cognitivo, motor e inclusive social, devido às relações interpessoais; impacta, portanto, no aumento do autoconceito dessa população, como mostram os estudos de Blomfield e Barber (2009) e de Liu et al. (2015).

Para Guimarães et al. (2020), o futebol é um fenômeno sociocultural no Brasil, compreendendo a imensa relevância no cotidiano da sociedade. Nesse sentido, é percebida uma alta demanda de praticantes com diferentes objetivos, que vão do recreativo até o alto rendimento. Para estes autores, é inquestionável que dentre os esportes coletivos o futebol é o preferido das crianças brasileiras do gênero masculino.

Ser um atleta é uma construção desenvolvida ao longo da vida, resultante de meios como as relações sociais (família, escola, técnicos, colegas), experiências em competições e treinamentos diários (Nascimento et al., 2016), tornando-se necessário cultivar e desenvolver um ambiente saudável e de bons relacionamentos entre os indivíduos do grupo.

Nesse sentido, o presente estudo investigou como os temas sobre a autoeficácia e o autoconceito têm sido pesquisados no futebol com jovens atletas, pois, como afirma (Wolf, 2015), a autoeficácia pode aumentar ou diminuir a motivação, no caso do esporte e do desenvolvimento dos adolescentes; quando positiva ela tende a aumentar a motivação, mas, caso seja negativa, geram-se dúvidas em relação à capacidade de resolver os problemas. O objetivo deste trabalho é investigar quantos estudos estão relacionando o autoconceito e a autoeficácia com a prática do futebol em jovens atletas.

\section{Metodologia}

O presente trabalho é um estudo de Scoping Review, conforme o método de revisão proposto pelo Instituto Joanna Briggs (Aromataris \& Munn, 2020). Este método permite identificar os tipos de evidências disponíveis em um determinado campo ou identificar as principais características ou fatores relacionados a um conceito. Para a construção da pergunta de pesquisa, utilizou-se a estratégia Population, Concept e Context (PCC) para uma scoping review. Foram definidos: P - Jovens atletas; C - Autoeficácia e Autoconceito; C - Futebol. Com base nessas definições foram estabelecidas as perguntas norteadoras: 1) Quais artigos científicos relacionam a autoeficácia ou o autoconceito com o futebol ou outras modalidades esportivas? 2) Quais são as populações estudadas nesses artigos e em qual país foi desenvolvida a pesquisa?

Para sistematizar a busca dos materiais, foram eleitas as bases de dados indexadas: Periódicos Eletrônicos em Psicologia - Pepsic; Periódicos da CAPES; Educational Resources Information Center - ERIC; SportDiscus; Biblioteca Virtual da Saúde - BVS; PsychINFO, pesquisadas na semana de 18 a 24 de julho de 2021, considerando os descritores 'selfefficacy', 'self-concept', 'young athletes', 'soccer' e 'collective sports', usando os operadores boleanos 'AND' e 'OR'. Foram considerados todos os tipos de pesquisa.

Para os critérios de inclusão foram considerados estudos que apresentam a relação da autoeficácia ou do autoconceito em jovens atletas de futebol a partir de 2011. Foram excluídos os estudos anteriores a 2011, duplicidade em diferentes bases, bem como estudos relacionados a outro tipo de população ou contexto.

Foram idealizadas três fases distintas para a meta de seleção dos estudos: (1) fase de buscas, em que foram coletados os estudos nas bases indexadas; (2) fase de seleção, que tem por objetivo filtrar os documentos, considerando os critérios 
propostos de inclusão e duplicidades apuradas; e (3) fase de eleição, na qual foram definidos os estudos para a composição dos quadros de análise que temos a seguir (Figura 1).

Figura 1. Visualização das fases de busca, seleção e eleição dos artigos.

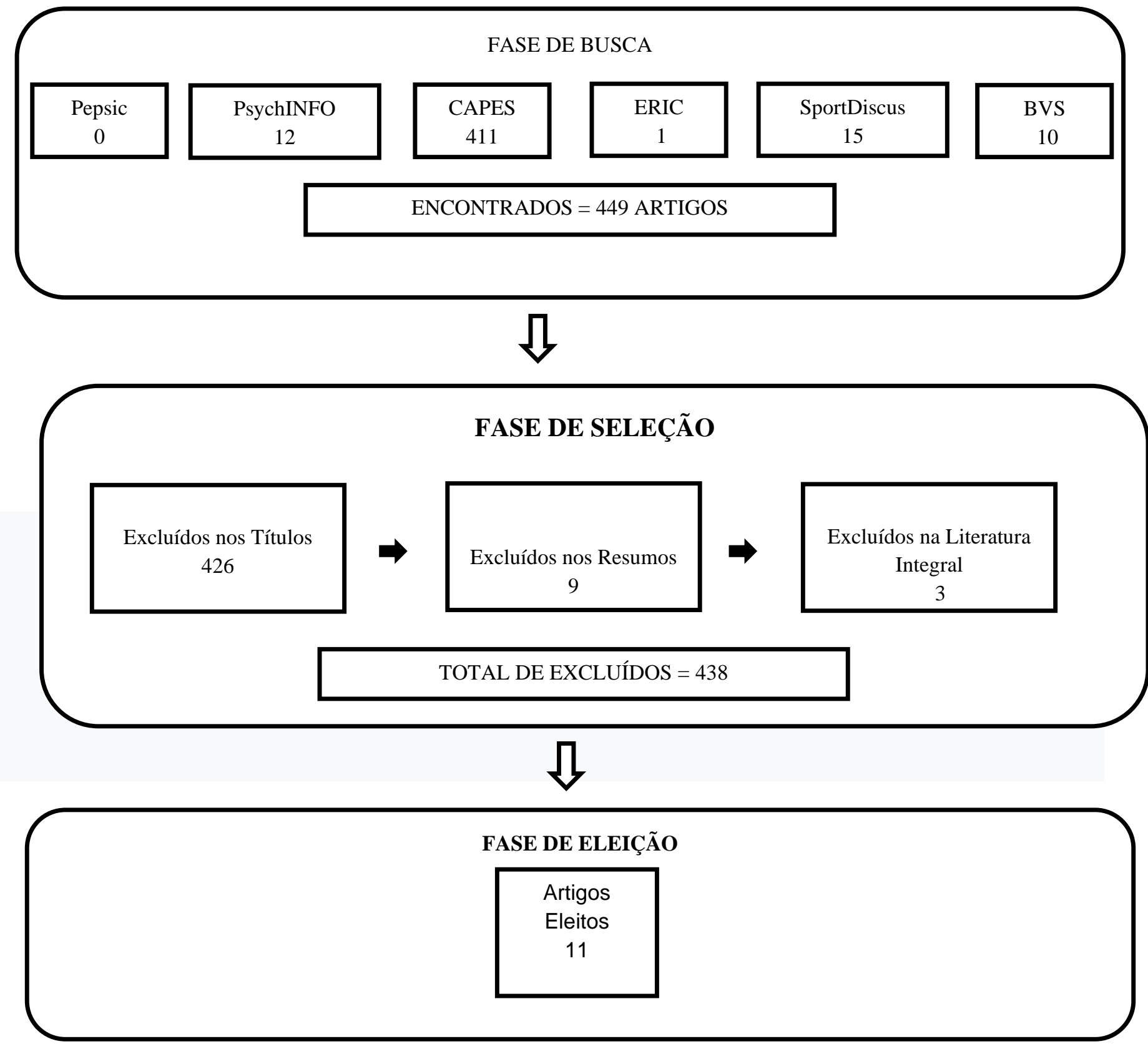

Fonte: Corrêa et al. (2022).

A divisão dos materiais para análise respeitou duas áreas de estudo: Autoeficácia (AE), Autoconceito (AC) e Futebol; Autoeficácia (AE), Autoconceito (AC) e outras modalidades para além do futebol. Após o processo de seleção, 11 estudos foram eleitos para análise (Tabela 1). 
Research, Society and Development, v. 11, n. 2, e21211225753, 2022

(CC BY 4.0) | ISSN 2525-3409 | DOI: http://dx.doi.org/10.33448/rsd-v11i2.25753

Tabela 1. Artigos eleitos por área.

\begin{tabular}{cc}
\hline TEMA & ELEITOS \\
\hline AE, AC e Futebol & $\mathbf{8}$ \\
AE, AC e Outras Modalidade & $\mathbf{3}$ \\
\hline \hline TOTAL & $\mathbf{1 1}$ \\
\hline \hline
\end{tabular}

Fonte: Corrêa et al. (2022).

\section{Resultados e Discussão}

A pesquisa analisou 11 artigos que foram organizados em: título; autor/data; país; objetivo; modalidades estudadas, apresentados em ordem decrescente a partir do ano de publicação. (Quadro 1)

A apreciação qualitativa dos artigos foi ordenada pela distinção das publicações, sendo: título, autor/data, tipo de estudo, país, objetivos, modalidades estudadas. A pesquisa analisou 9 artigos, organizados no quadro a seguir: 
Research, Society and Development, v. 11, n. 2, e21211225753, 2022

(CC BY 4.0) | ISSN 2525-3409 | DOI: http://dx.doi.org/10.33448/rsd-v11i2.25753

Quadro 1. Descrição dos artigos em ordem decrescente do ano de sua publicação.

\begin{tabular}{|c|c|c|c|c|}
\hline TÍTULO & AUTOR/DATA & PAÍS & OBJETIVO & MODALIDADES \\
\hline $\begin{array}{l}\text { Differences in the psychological } \\
\text { profiles of elite and non-elite } \\
\text { athletes }\end{array}$ & $\begin{array}{c}\text { Mitic; } \\
\text { Nedelijkovic; } \\
\text { Bojanic; } \\
\text { Francesco; } \\
\text { Milavanociv; } \\
\text { Bianco; } \\
\text { Drid. } \\
\text { (2021) }\end{array}$ & $\begin{array}{l}\text { República } \\
\text { Da Sérvia }\end{array}$ & $\begin{array}{l}\text { Determinar se o conjunto de características psicológicas } \\
\text { específicas (autoeficácia, perspectiva de tempo, inteligência } \\
\text { emocional, motivação de realização e dimensões de } \\
\text { personalidade) fazem a distinção entre atletas com base na sua } \\
\text { participação ou não na seleção nacional }\end{array}$ & $\begin{array}{c}\text { Futebol de campo } \\
\text { Basquete } \\
\text { Vôlei } \\
\text { Judô } \\
\text { Polo Aquático } \\
\text { Handebol }\end{array}$ \\
\hline $\begin{array}{l}\text { Using self-assessment to build } \\
\text { self-efficacy and intrinsic } \\
\text { motivation in athletes: a mixed } \\
\text { methods explanatory design on } \\
\text { female adolecent volleyball } \\
\text { players }\end{array}$ & $\begin{array}{l}\text { Knight } \\
(2020)\end{array}$ & $\begin{array}{l}\text { Estados } \\
\text { Unidos }\end{array}$ & $\begin{array}{l}\text { Este estudo de métodos mistos abordou a questão do burnout e } \\
\text { a falta de motivação em alunos-atletas do ensino fundamental e } \\
\text { médio utilizando um modelo que foi projetado para usar a } \\
\text { autoavaliação e aumentar a autoeficácia entre os atletas }\end{array}$ & Vôlei \\
\hline $\begin{array}{l}\text { Testing a brief coping intervention } \\
\text { for high-performance youth soccer } \\
\text { players and their parents }\end{array}$ & $\begin{array}{l}\text { Tamminen; Pennock; } \\
\text { Braun. } \\
\text { (2019) }\end{array}$ & Canadá & $\begin{array}{c}\text { Implementar uma oficina de enfrentamento para jovens atletas } \\
\text { e que também envolvesse os pais para fornecer-lhes mais } \\
\text { informações para apoiar as habilidades de enfrentamento em } \\
\text { seus filhos e perceber o quanto isso eleva sua percepção de } \\
\text { autoeficácia }\end{array}$ & Futebol de campo \\
\hline $\begin{array}{l}\text { Perceptions of parenting practices } \\
\text { and psychological variables of elite } \\
\text { and sub-elite youth athletes }\end{array}$ & $\begin{array}{c}\text { Teques; } \\
\text { Calmeiro; } \\
\text { Rosado; } \\
\text { Siilva; } \\
\text { Serpa. } \\
(2019) \\
\end{array}$ & Portugal & $\begin{array}{l}\text { Examinar as associações de percepções das práticas parentais } \\
\text { (incentivo, reforço, instrução e modelagem de papéis) e } \\
\text { variáveis psicológicas dos atletas (autoeficácia, autoeficácia } \\
\text { social, autorregulação e motivação intrínseca) da elite e atletas } \\
\text { jovens de sub-elite }\end{array}$ & $\begin{array}{l}\text { Futebol de campo } \\
\text { Basquete } \\
\text { Vôlei } \\
\text { Handebol }\end{array}$ \\
\hline $\begin{array}{c}\text { Influencia del apoyo } \\
\text { socioemocional sobre la } \\
\text { afectividad experimentada, } \\
\text { autoconfianza y autoeficacia em } \\
\text { jóvenes desportistas }\end{array}$ & $\begin{array}{l}\text { López; } \\
\text { Granado; } \\
\text { Lira; } \\
\text { Cerrato } \\
\text { (2018) }\end{array}$ & Chile & $\begin{array}{l}\text { Comprovar através de um modelo de equação estrutural, a } \\
\text { influência das relações de apoio a autonomia do esportista } \\
\text { sobre sua afetividade positiva e regulação emocional } \\
\text { experimentada em competições, e a aquisição de recursos } \\
\text { pessoais como a autoconfiança e a autoeficácia }\end{array}$ & $\begin{array}{c}\text { Futebol de campo, Canoagem, } \\
\text { Atletismo, Natação, } \\
\text { Ginástica rítmica, Tênis, } \\
\text { Basquete, } \\
\text { Vôlei } \\
\text { Rúgbi }\end{array}$ \\
\hline $\begin{array}{l}\text { The effects of coaches's pre-game } \\
\text { speeches on Young players' self- }\end{array}$ & $\begin{array}{l}\text { Rubio; Hernandes; Sanchez- } \\
\text { Iglesias; }\end{array}$ & Espanha & $\begin{array}{l}\text { Este estudo examina os efeitos do discurso dos técnicos sobre a } \\
\text { autoeficácia de jovens atletas em dois estudos quantitativos }\end{array}$ & Futebol de campo \\
\hline
\end{tabular}


Research, Society and Development, v. 11, n. 2, e21211225753, 2022

(CC BY 4.0) | ISSN 2525-3409 | DOI: http://dx.doi.org/10.33448/rsd-v11i2.25753

\begin{tabular}{|c|c|c|c|c|}
\hline efficacy & $\begin{array}{l}\text { Cano; } \\
\text { Bureo } \\
(2018)\end{array}$ & & diferentes & \\
\hline $\begin{array}{l}\text { Enhancing self-efficacy through a } \\
\text { blended training: a pilot study with } \\
\text { basketball players }\end{array}$ & $\begin{array}{c}\text { Villani } \\
\text { Caputo } \\
\text { Balzarotti } \\
\text { Riva } \\
(2017)\end{array}$ & Itália & $\begin{array}{l}\text { Neste estudo foi testada a eficácia de um programa de } \\
\text { treinamento inovador combinando atividades face a face e } \\
\text { baseadas na web para aumentar a autoeficácia e a regulação } \\
\text { emocional entre jovens jogadores de basquete }\end{array}$ & Basquete \\
\hline $\begin{array}{l}\text { The relationships between athletes' } \\
\text { perceptions of coach-created } \\
\text { motivational climate, self-talk, and } \\
\text { self-efficacy in youth soccer }\end{array}$ & $\begin{array}{c}\text { Zourbanos; } \\
\text { Haznadar; } \\
\text { Papaioannou; } \\
\text { Tzioumakis; } \\
\text { Krommidas; } \\
\text { Hatzigeorgiadis } \\
\quad(2016)\end{array}$ & Grécia & $\begin{array}{l}\text { Investigar o papel mediador do diálogo interno na relação entre } \\
\text { as percepções de jovens atletas sobre o clima motivacional } \\
\text { criado pelo técnico e as crenças de autoeficácia dos atletas }\end{array}$ & Futebol de campo \\
\hline $\begin{array}{l}\text { Athletes' perception of coaching } \\
\text { behavior, relation-inferred self- } \\
\text { efficacy, and self-efficacy in youth } \\
\text { sport }\end{array}$ & $\begin{array}{l}\text { Saville } \\
\text { Bray } \\
(2016)\end{array}$ & Canadá & $\begin{array}{l}\text { Investigar as associações entre os comportamentos do treinador } \\
\text { e a autoeficácia no esporte juvenil }\end{array}$ & $\begin{array}{l}\text { Futebol de campo } \\
\text { Hóquei no gelo } \\
\text { Ringette }\end{array}$ \\
\hline $\begin{array}{l}\text { Goal orientation and self-efficacy } \\
\text { as predictor of male adolescente } \\
\text { soccer players' motivation to } \\
\text { participate }\end{array}$ & $\begin{array}{l}\text { Çentinkalp } \\
\text { Tutksoy } \\
(2011)\end{array}$ & Turquia & $\begin{array}{c}\text { Determinar se a orientação para metas e o nível de autoeficácia } \\
\text { podem prever a motivação para participar de um jogo de } \\
\text { futebol com jovens atletas }\end{array}$ & Futebol de campo \\
\hline $\begin{array}{l}\text { Coping skills and self-efficacy as } \\
\text { predictor of gymnastic } \\
\text { performance }\end{array}$ & $\begin{array}{l}\text { Daroglou } \\
(2011)\end{array}$ & Grécia & $\begin{array}{c}\text { Investigar a forma como o desempenho da ginástica pode ser } \\
\text { discriminado com base em habilidades psicológicas e } \\
\text { autoeficácia }\end{array}$ & $\begin{array}{l}\text { Ginástica rítmica } \\
\text { Ginástica artística }\end{array}$ \\
\hline
\end{tabular}

Autores: Corrêa et al. (2022). 


\subsection{Relação da Autoeficácia em jovens atletas de futebol}

Oito artigos sobre a autoeficácia (Mitic et al., 2021; Tamminem et al., 2019; Teques et al., 2019; López et al., 2018; Rubio et al., 2018; Zourbanos et al., 2016; Saville; bray, 2016; Centinkalp; Tutksoy, 2011) foram encontrados relacionando sua temática ao futebol.

Todos os trabalhos apresentam uma forma específica de intervenção e investigação para determinar situações relacionadas à autoeficácia em atletas de futebol. Dois deles foram realizados no Canadá (Tamminem et al., 2019; Saville; Bray, 2016), um no Chile (López et al., 2018), um na Espanha (Rubio et al., 2018), um na Sérvia (Mitic et al., 2021), um em Portugal (Teques et al., 2019), um na Grécia (Zourbanos et al., 2016) e um na Turquia (Centinkalp \& Tutksoy, 2011).

Os estudos apresentam focos diversificados de investigação, variando em: comprovar a influência das relações de apoio à autonomia do esportista sobre sua afetividade positiva e regulação emocional experimentada em competições (López et al., 2018); examinar os efeitos do discurso dos técnicos sobre a autoeficácia dos atletas (Rubio et al., 2018); implementar uma oficina de enfrentamento para jovens atletas e perceber o quanto isso eleva sua percepção de autoeficácia (Tamminem et al., 2019); determinar se o conjunto de características psicológicas específicas faz a distinção entre atletas com base na sua participação ou não na seleção nacional (Mitic et al., 2021); examinar as associações de percepções das práticas parentais e variáveis psicológicas dos atletas da elite e atletas jovens de sub-elite (Teques et al., 2019).

Além destes, (Zourbanos et al. 2016) investigaram o papel mediador do diálogo interno na relação entre as percepções de jovens atletas sobre o clima motivacional criado pelo técnico e suas crenças de autoeficácia. (Saville \& Bray, 2016) investigaram as associações entre os comportamentos do treinador e a autoeficácia no esporte juvenil. Por fim, Centinkalp e Tutksoy, (2011) determinaram se a orientação para metas e o nível de autoeficácia podem prever a motivação para participar de um jogo de futebol com jovens atletas.

Nesses artigos, apresentamos uma série de pesquisas que envolvem a percepção da autoeficácia de jovens atletas de futebol e diversas formas de como essa questão foi abordada. É interessante notar que os estudos mostram vários elementos que fazem parte do contexto esportivo, como a família e os treinadores, peças-chave para o sucesso desportivo do atleta.

Os trabalhos não possuem uma forma de investigação única, o que mostra como este tema pode ser observado e analisado de diversas formas: revisão de escopo, revisão sistemática, bibliométrica. Portanto, no futebol, a autoeficácia pode ser estudada de diversas formas, o pesquisador pode se deparar com uma demanda específica, perceber a melhor forma de ir atrás das suas hipóteses e compreender quais os melhores caminhos para encontrar documentos que sejam pertinentes a este campo de estudo.

\subsection{Relação da Autoeficácia em jovens atletas de outras modalidades}

Três artigos sobre a autoeficácia (Daroglou, 2011; Villani et al., 2017; Knight, 2020) foram encontrados relacionando sua temática com outras modalidades, com o propósito de comparar se os estudos externos ao contexto do futebol envolviam as mesmas investigações. Todos os trabalhos apresentam uma forma específica de intervenção, de investigação, para determinar situações relacionadas à autoeficácia em atletas de outras modalidades, como voleibol, basquetebol, ginástica artística e rítmica (Daroglou, 2011; Villani et al., 2017; Knight, 2020).

Com relação à modalidade e ao país de origem dos estudos, o trabalho de Knight (2020) foi realizado com o voleibol nos Estados Unidos, o trabalho de (Villani et al., 2017) foi realizado na Itália com atletas de basquetebol e o de (Daroglou, 2011), com atletas da ginástica rítmica e artística na Grécia.

Sobre às investigações dos estudos, (Knight, 2020) por meio de métodos mistos, utilizou a questão do burnout e a falta de motivação em alunos-atletas do ensino fundamental e médio com um modelo que foi projetado para usar a 
Research, Society and Development, v. 11, n. 2, e21211225753, 2022

(CC BY 4.0) | ISSN 2525-3409 | DOI: http://dx.doi.org/10.33448/rsd-v11i2.25753

autoavaliação e aumentar a autoeficácia entre os atletas de vôlei. (Villani et al.,2017) testaram a eficácia de um programa de treinamento combinando atividades face a face e atividades baseadas na web para aumentar a autoeficácia e a regulação emocional entre jovens jogadores de basquete. Por último, (Daroglou, 2011) investigou a forma como o desempenho da ginástica pode ser discriminado com base em habilidades psicológicas e na autoeficácia.

\section{Conclusão}

Este scoping review nos auxiliou a verificar possibilidades de estudos abarcados pela temática da autoeficácia no esporte, mais especificamente no futebol. Dentro desta revisão, tivemos acesso a trabalhos que foram voltados aos atletas de forma direta, com treinamentos e técnicas para potencializar sua autoeficácia.

Outros pesquisadores (Saville \& Bray, 2016; Rubio et al., 2018) realizaram seus estudos com treinadores para constatar o quanto seu papel interfere, positiva ou negativamente, na autoeficácia de seus atletas. Por fim, também temos alguns estudos (Teques et al., 2019) em que a investigação é realizada com os pais dos atletas, peças-chave no desenvolvimento esportivo dos seus filhos. Há ainda estudos que investigam o quanto sua influência impacta a autoeficácia dos filhos no ambiente esportivo.

Percebemos uma diversificação no tipo de estudo realizado pelos autores selecionados para a análise. No entanto, esta revisão teve por objetivo investigar os estudos mais recentes sobre a temática principal e perceber o quanto ela pode ser multifacetada e envolver vários agentes que participam do ambiente esportivo.

Com relação ao autoconceito, não foram encontrados estudos que se inserissem na temática proposta relacionando esse fator com jovens atletas de futebol, uma lacuna científica importante que abre caminho para novas investigações neste campo.

\section{Agradecimentos}

Agradecemos aos orientadores por todo aprendizado que nos proporcionaram junto a Universidade Federal do Paraná, setor de Educação, linha de Cognição, Aprendizagem e Desenvolvimento Humano em conjunto da equipe de professores.

Financiamento. O presente trabalho foi realizado com apoio da Coordenação de Aperfeiçoamento de Pessoal de Nível Superior (CAPES/ PROEX/ CNP).

\section{Referências}

Aromataris, E. M. (2020.). JBI Reviewer's Manual. JBI. 10.11124/JBISRIR-D-19-0009

Bandura, A., Freeman, W. H., \& Lightsey, R. (1999). Self-efficacy: The exercise of control. 10.1891/0889-8391.13.2.158

Blomfield, C. J., \& Barber, B. L. (2009.). Brief report: Performing on the stage, the field, or both? Australian adolescent extracurricular activity participation and self-concept. Journal of Adolescenc, 32(3), 733-739. doi.org/10.1016/j.adolescence.2009.01.003

Cetinkalp, Z. K., \& Turksoy, A. (2011). Goal orientation and self-efficacy as predictors of male adolescent soccer players' motivation to participate. Social Behavior and Personality: An international journal, 39(7), 925-934. https://doi.org/10.2224/sbp.2011.39.7.925

Coalter, F. (2013). 'There is loads of relationships here': Developing a programme theory for sport-for-change programmes. International review for the sociology of sport, 48(5), 594-612. https://doi.org/10.1177/1012690212446143

Gallagher, M. W., \& Ramachandran, V. S. (2012). Self-Efficacy. In: (Ed.). Encyclopedia of Human Behavior (Second Edition). San Diego: Academic Press(2), p. 314-320, 2012. doi:10.1177/155982761349806139.

Guimarães, M. B., De Oliveira, A. M., \& Paoli, P. B. (2020). A prospecção do talento no futebol brasileiro:: diagnóstico estrutural e financeiro do processo de captação de atletas ( $1^{\mathrm{a}}$ ed.). Curitiba: Appris. Retrieved january 01,2022 from https://shortest.link/2FVh

Knight, A. (2020). Using Self-Assessment to Build Self-Efficacy and Intrinsic Motivation in Athletes: A Mixed Methods Explanatory Design on Female Adolescent Volleyball Players. Qualitative Report, 25(2a) Retrieved january 01,2022 from. https://shortest.link/2FV8 
Research, Society and Development, v. 11, n. 2, e21211225753, 2022

(CC BY 4.0) | ISSN 2525-3409 | DOI: http://dx.doi.org/10.33448/rsd-v11i2.25753

Kyrillos, M. H. (2016). Motivos para a prática esportiva e o senso de autoeficácia em atletas. (Dissertação (Mestrado em Educação Física) - Universidade Federal de Sergipe, Aracaju, 2016. Retrieved january 01,2022 from. https://ri.ufs.br/jspui/handle/123456789/4966

Liu, M., Wu, L., \& Ming, Q. (2015). How Does Physical Activity Intervention Improve Self-Esteem and Self-Concept in Children and Adolescents? . Evidence from a Meta-Analysis. PloS One, 10(8), 1-17.https://doi.org/10.1371/journal.pone.0134804

López, V. M. (2018). Influencia del apoyo socioemocional sobre la afectividad experimentada, autoconfianza y autoeficacia em jóvenes desportistas. Revista de Psicología del Deporte/ Journal of Sport Psychology, 1, 51-58. Retrieved january 01,2022 from https://shortest.link/2yIb

Matias, T. S. (2009). Estilo de vida, nível habitual de atividade física e percepção de autoeficácia de adolescentes. Revista da Educação Física/UEM, 20(2a), 235-243. 10.4025/reveducfis.v20i2.6413

Mitić, P. (2021). Differences in the Psychological Profiles of Elite and Non-elite Athletes. Frontiers in Psychology , 12, 769. https://doi.org/10.3389/fpsyg.2021.635651

Monteiro, V. (2012). Promoção do autoconceito e autoestima através de um programa de leitura a par. Psicologia: Reflexão e Crítica, 25(1'), 147-155. https://doi.org/10.1590/S0102-79722012000100018

Nascimento, M. G. (2016). Psychological profiles of gender and personality traces os Brazilian professional atlhetes of futsal, and their infuence on physiological parameters. Psychology Research and Behavior Management, 41-51. 10.2147/PRBM.S77402

Reverdito, R. (2016). Pedagogia do esporte e modelo bioecológico do desenvolvimento humano: indicadores para avaliação de impacto em programa socioesportivo. 2016. 209 f. Tese (Doutorado em Educação Física) - Universidade Estadual de Campinas, Campinas. Retrieved january 01,2022 from https://shortest.link/2FVF

Rubio, V. J. (2018). The effects of coaches’ Pre-game on Young players' self-efficacy. . Revista de Psicología del Deportel Journal os Sport Psychology, 27( $\left.1^{\text {a }}\right), 59-66$. . Retrieved january 01,2022 from https://shortest.link/2FVK

Sarriera, J. C. (2015). Propriedades psicométricas da Escala de Autoconceito Multidimensional em adolescentes brasileiros. Avaliação Psicológica, 14(2 ${ }^{\mathrm{a}}$ ), 281-290. 10.15689/ap.2015.1402.13

Saville, D. P., \& Bray, S. R. (2016). Athletes' perceptions of coaching behavior, relation-inferred self-efficacy (RISE),and self-efficacy in youth sport. Journal of Applied Sport Psychology, 28, 1-13. https://doi.org/10.1080/10413200.2015.1052890

Serra, A. S. (1988). O auto-conceito. Análise Psicológica, 6(2a), 101-110. Retrieved january 01,2022 from https://core.ac.uk/download/pdf/70651471.pdf

Stevanato, I. S. (2003). Autoconceito de crianças com dificuldades de aprendizagem e problemas de comportamento. . Psicologia em Estudo, 8(1 ${ }^{\text {a }}$, 67-76. https://doi.org/10.1590/S1413-73722003000100009

Tamayo, A. (2001). A influência da atividade física regular sobre o autoconceito. Estudos de Psicologia, 6(2a), 157-165. https://doi.org/10.1590/S1413294X2001000200004

Tamminen, K. A. (2019). Testing a brief coping intervention for high-performance youth soccer players and their parents. Case studies in Sport and Exercise Psychology, 3(1 $\left.{ }^{\text {a }}\right)$, 61-70. https://doi.org/10.1123/cssep.2019-0009

Teques, P. (2019). Perceptons of parenting practices and psychological variables of elite and sub-elite youth athletes. Frontiers in Psychology, 10. https://doi.org/10.3389/fpsyg.2019.01495

Villani, D. (2017). Enhancing self-efficacy through a blended training: a pilot study with basketball players. International Journal of Sport Psychology, 15(2 ${ }^{\text {a }}$, 160-175.https://doi.org/10.1080/1612197X.2015.1079921

Wolf, A. A. (2015). Pensamento Campeão: melhorando o desempenho esportivo por meio da preparação mental: um gruia de psicologia do esporte baseado na terapia cognitivo-comportamental. Rio de Janeiro: Editora Cognitiva. Retrieved january 01,2022 from https://shortest.link/2FW4

Zourbanos, N. (2016). The relationships between athletes' perceptions of coach-created motivacional climate, self-talk, and self-efficacy in youth soccer. Journal of Applied sport psychology, 28, 97-112. https://doi.org/10.1080/10413200.2015.1074630 